\title{
Fluorescent Molecule Incorporated Metal-Organic Framework for Fluoride Sensing in Aqueous Solution
}

\author{
Xudong Zhao*, Yuanyang Wang, XiuliHao, Wen Liu* \\ College of Chemical and Biological Engineering
}

Taiyuan University of Science and Technology, Taiyuan 030012, China

Corresponding Authors E-mail: zhaoxd_tykj@163.com; 13700509372@163.com 


\section{Abstract}

In this work, the fluorescent molecule (fluorescein sodium, FS) was successfullyincorporated in the zirconium-based MOF (UiO-66) via in-situ synthesis method, which can be confirmed by FTIR spectra and fluorescence microscopic images.Based on this in-situ synthesis strategy, FS molecule can be immobilized tightly in the framework. Furthermore, the resulting FS@UiO-66 demonstrates to be a highly selective, real-time and naked-eye chemical sensor for fluoride in aqueous solution, which is mainly due to the release of FS molecule from FS@UiO-66 into the aqueous solution. Meanwhile, to the best of our knowledge, suchZr-MOF-based fluoride sensoris very rare so far. These results provide a promising approach to rationally design novel MOF-based fluorescent sensor for the target molecules.

Keywords: Fluorescent molecule; Metal-organic framework; Fluorescence sensing; Fluoride 


\section{Introduction}

As one of the human body essential trace elements, fluoride plays an important role in dental and sclerotin health. However, excessive fluoride in water can lead to kinds of health problems such as softening of bones, neurological disorder and kidney problems[1-2]. Therefore, the detection for fluoride in water is of great importance to improve the public health. Compared to the traditional methods, fluorescence detection method attracts large attention due to its advantages such as high selectivity, quick response and naked-eye sensingability [1].

Metal-organic frameworks (MOFs), as a novel class of microporous crystalline materials, have been widely studied in many fields[3-9]. In particular, owing to theremarkable features including abundant $\pi$-conjugated system, controllable framework structure and binding sites, MOFs have exhibitedpotential applications in chemical sensing[10-11]. As an indispensable part of MOF-based fluorescent sensor, chromophore has been successfully constructed through proper selection of luminescent parts such as organic molecules[12-15]. In previous works, several organic fluorescence molecules have been encapsulated into ZJU-88[12], Mn-NDC[13], ZIF-8[16] and Zn(bix)[17]by in-situ synthesis for effectively sensingtemperature, $\mathrm{SO}_{4}{ }^{2-}$, aniline and water/ethanol, respectively. However, relevant attempts have rarely been performed to design MOF sensors for fluoride, let aloneZr-based MOFs.

UiO-66, consisting of zirconium ion and 1,4-benzenedicarboxylic acid $\left(\mathrm{H}_{2} \mathrm{BDC}\right)$, has great potential in many fields such as adsorption[18-19], separation[20] and 
catalysis[21]. However, the fixation capacity for organic fluorescence molecules in UiO-66 and relevant chemical sensors have rarely been reported so far [22]. Herein, fluorescein sodium hosted in UiO-66 was prepared and well characterized by powder X-ray diffraction (PXRD), $\mathrm{N}_{2}$ adsorption, FTIR spectra and fluorescence microscopic images. Interestingly, the resulting FS@UiO-66 is capable of highly selective detection of fluoride with a short response time.

\section{Materials and methods}

\subsection{Materials synthesis}

UiO-66[23]. 1,4-benzenedicarboxylic acid (0.08 g) and $\mathrm{ZrCl}_{4}(0.12 \mathrm{~g})$ were mixed in DMF (20 mL). After stirred for $10 \mathrm{~min}$, the mixture was transferred into a $100 \mathrm{~mL}$ Teflon lined autoclave and then heated at $393 \mathrm{~K}$ for $22 \mathrm{~h}$. After being cooled down to room temperature, the white solid was obtained through filtration and washing with DMF and ethanol several times. At last, the solid was dried under vacuum at $373 \mathrm{~K}$ for $12 \mathrm{~h}$.

FS@UiO-66. 1,4-benzenedicarboxylic acid (0.08 g), $\mathrm{ZrCl}_{4}(0.12 \mathrm{~g})$ and fluorescein sodium (0.03 g, the molecular structure is shown in Fig. 1$)$ were mixed in DMF (20 $\mathrm{mL}$ ). The reaction process was similar to that of UiO-66. After being cooled down to room temperature, the obtained yellow solid was filtered and washed with DMF and ethanol until the fluorescence of the washing solvent is almost invisible. At last, the yellow solid was also heated under vacuum at $373 \mathrm{~K}$ for $12 \mathrm{~h}$.

\subsection{Characterization techniques}

All the chemicals in the experiments were commercially available and used without 
further purification. Powder X-ray diffraction (PXRD) of the materials were measured on a D8 Advance $\mathrm{X}$ diffractometer with $\mathrm{Cu} \mathrm{K} \alpha$ radiation $(\lambda=1.5406 \AA$ ). The BET surface areas of MOFs were measured on an Autosorb-IQ-MP surface area analyzer using $\mathrm{N}_{2}$ adsorption at $77 \mathrm{~K}$. The infrared (IR) spectra were recorded on a Nicolet 6700 Fourier Transform Infrared Spectrometer with the $\mathrm{KBr}$ pellet technique. Photoluminescence (PL) spectra were collected using an F-7000 fluorescence spectrometer with blazed holographic concave diffraction grafting and a xenon lamp was used as the excitation source. X-ray photoelectron spectroscopy (XPS) was measured on a PerkinElmer PHI-5400 photoelectron spectrometer. Fluorescence microscopic (FM) images were collected on an Olympus IX73 FM.

\subsection{Fluorescence experiments}

In the fluorescence experiments towards anions, $10 \mathrm{mg}$ MOF sample was added into $10 \mathrm{~mL}$ aqueous solutions containing different anions with certain concentrations. After shaking for a short time, the suspension was aged for the certain time. Then the upper solution was extracted using a syringe and filtered by the microfiltration membrane. The clear filter liquor was collected and used in the measurement of fluorescence intensity.

In the experiments towards fluoroorganics, $10 \mathrm{mg}$ MOF sample was added into 10 $\mathrm{mL}$ DMF solutions containing different fluoroorganic species (concentration is $10^{-2}$ M). The subsequent process is similar to the process above.

\section{Results and discussion}

\subsection{Characterization of materials}


To confirm the existence form of FS molecule in FS@UiO-66, energy dispersive X-ray (EDX) measurement was performed. The EDX spectrum of the synthesized sample reveals that the sample composition is $\left(\mathrm{C}_{3.35} \mathrm{O}_{1.7} \mathrm{Na}_{0.097} \mathrm{Zr}_{0.33}\right)_{\mathrm{m}}$. Based on the fact that the elemental composition of FS and UiO-66 are $\mathrm{C}_{20} \mathrm{H}_{10} \mathrm{Na}_{2} \mathrm{O}_{5}$ and $\mathrm{C}_{24} \mathrm{H}_{14} \mathrm{O}_{16} \mathrm{Zr}_{3}$ respectively, the composition of the synthesized sample is calculated to be about $(0.049 \mathrm{FS} \cdot 0.11 \mathrm{UiO}-66)_{\mathrm{n}}$. Therefore, we suggest that the whole FS molecule enters the composite.

Fig. 2a shows that the patterns ofsynthesized FS@UiO-66 and UiO-66 are similar to simulated pattern of UiO-66, except for the diffraction peak at $12^{\circ}$ which is attributed to the existence of the solvent [24]. This indicates that FS@UiO-66 has similar topology structure with UiO-66. To verify the permanent porosity of FS@UiO-66, $\mathrm{N}_{2}$ adsorption-desorption isothermals at $77 \mathrm{~K}$ were further performed.As shown in Fig. 2b,the BET surface area of FS@UiO-66 is evaluated to be $641 \mathrm{~m}^{2} / \mathrm{g}$, far lower than that of the parent UiO-66. This significant decrease is mainly attributed to the weaker crystallinity or the amorphous nature of some pore walls[25-26].

Verification of existence of FS molecule was conducted through FTIR spectra and fluorescence microscopy images. The bands observed at $1018 \mathrm{~cm}^{-1}$ can be assigned to the $\mathrm{C}-\mathrm{O}$; the bands observed at $1638 \mathrm{~cm}^{-1}$ and $1643 \mathrm{~cm}^{-1}$ can be assigned to the $\mathrm{C}=\mathrm{O}$ in benzoquinone structure of FS molecule (this special structure in FS does not exist in UiO-66) rather than in -COO structure $\left(1590 \mathrm{~cm}^{-1}\right)$ [27]. From Fig. 3, it can be seen that after FS is loaded, the peak at $1018 \mathrm{~cm}^{-1}$ becomes strong and the peak at 
$1643 \mathrm{~cm}^{-1}$ appears.Furthermore, as shown in Figs. 4a and 4b, almost all the MOF particles can emit bright yellowish green fluorescence under the UV light of $365 \mathrm{~nm}$. These results indicate that FS molecule has been encapsulated into UiO-66 during the formation process of the framework [28].

In addition, previous work has explained that the peak $\left(550 \mathrm{~cm}^{-1}\right)$ in the spectrum of UiO-66 is attributed to the asymmetric stretching vibration of $\mathrm{Zr}-(\mathrm{OC})$ [27]. In this work, the peak of Zr-(OC) after FS molecule is incorporated turns slightly from 550 $\mathrm{cm}^{-1}$ to $539 \mathrm{~cm}^{-1}$ (Fig. 3), which indicates that the encapsulation of FS molecule has little effect on the coordination mode between $\mathrm{Zr}^{4+}$ and $\mathrm{H}_{2} \mathrm{BDC}$ in the framework[17]. This unobvious shift is mainly attributed to the weak interaction between FS molecule and UiO-66 in the in-situ self-assembly process[17].

\subsection{Luminescent properties}

UiO-66, as one of the classical MOFs, was rarely reported in the luminescence field for its poor luminescence performance due to ligand to metal charge transform (LMCT) process[29]. However, thanks to excellent stability and special topology structure, UiO-66 or modified UiO-66 provides an excellentplatform to load organic molecule to construct novel chemical sensor viapostsyntheticmethod (PSM) [22, 30].In this work, to study the potential of UiO-66 in sensing fluoride, we tried to encapsulate FS molecule into thisZr-based frameworkviain-situ synthesis method.As illustrated in Fig.5a, FS@UiO-66 showsstrong emission peak centered at 537 nm excited at $490 \mathrm{~nm}$. Furthermore, to confirm the tight immobilization of FS molecule in the resulting material, FS@UiO-66 was immersed in pure water with different time 
and the corresponding solid-state PL spectra were measured after being filtered and dried. As depicted in Fig. 5b, long-time (24 h) immersion in pure water has negligible effect on the fluorescence intensity of FS@UiO-66, indicating that this synthesis strategy is effective for the stable encapsulation of the organic molecule.

\subsection{Sensing performance}

To investigate the sensing performance for anions, FS@UiO-66 sample was immersed in aqueous solutions containing different anions $\left(\mathrm{NO}_{3}{ }^{-}, \mathrm{SO}_{4}{ }^{2-}, \mathrm{OAc}^{-}, \mathrm{F}^{-}, \mathrm{Cl}^{-}\right.$, $\mathrm{Br}^{-}, \mathrm{I}^{-}$) for some time and then the upper clear solution was selected for the measurement of PL intensity. As shown in Fig.6a, the colorless fluoride solution turns to yellow solution after $12 \mathrm{~h}$ under natural light and shows bright yellowish green fluorescence under UV light at $365 \mathrm{~nm}$ (Fig. 6b). Meanwhile, there is an obvious emission peak centered at $520 \mathrm{~nm}$ excited at $490 \mathrm{~nm}$ in the PL spectrum of the fluoride solution (Fig.6c). However, other anions cannot induce the similar phenomenon. Furthermore, the effect of co-exist anions on the sensing performance of FS@UiO-66 was also investigated. FS@UiO-66 sample was immersed in the ion-mixed solutions and the PL spectra for the upper clear solutions were measured. As shown in Fig. 7, other anions have negligible effect on the recognition for fluoride. These results demonstrate that FS@UiO-66 exhibits excellent selectivity in fluoride sensing. In addition, it is noted that the yellow solid (FS@UiO-66) in the bottom has diminished in fluoride solution, which will be discussed in detail in the following part of this article.

To investigate the time-response performance of FS@UiO-66 toward fluoride, the 
PL intensity of the upper clear solution at $520 \mathrm{~nm}$ was measured as a function of immersion time in aqueous solutions of fluoride $\left(10^{-2} \mathrm{M}\right)$. As the time-response curve shownin Fig. 8a, it is observed that the fluorescence intensity of the solution increases sharply in 5 min and reachesthe maximum value at 15 min. Meanwhile, there is an obvious increase of the intensity within seconds, which indicates that the fluoride-induced fluorescence response is very fast[1].

To better understand the sensing performance for fluoride of FS@UiO-66, the PL intensities were also measured after being immersed in fluoride solutions with different concentrations for 24 h. As indicated in Fig. 8b, the emission intensity of upper clear solution increases in accordance with the increase of the fluoride concentration from 0 to $10^{-1}$ M.In addition, when the fluoride concentration increases from 0 to $0.4 \mathrm{mM}$, a good linear relationship $\left(R^{2}=0.99885\right)$ between the PL intensity and the concentration of fluoride is observed. The detection limit of FS@UiO-66 towards fluoride is calculated to be $4.4 \times 10^{-3} \mathrm{mMaccording}$ to the literature [31]. From Table 1, (a) the detection limit of FS@UiO-66 is higher than that of some organic compounds such as 4-bromo-2,6-bis(hydroxymethyl)phenol, aminophenylpyridinium and BODIPY dye-containing chemodosimeter [32-34], however, FS@UiO-66 can detect fluoride in aqueous solution rather than in organic solvent, which is more important to improve the public health; (b) the detection limit of FS@UiO-66 is much lower than that of some quantum dots (QD) such as CdSe/ZnS QD [35]; (c) in MOF-based sensors, the detection limit of FS@UiO-66 is a little higher than that of $\left[\mathrm{Cu}_{4} \mathrm{I}(\mathrm{TIPE})_{3}\right] \cdot 3 \mathrm{I}$ [36], however, the fluorescence “turn-on” FS@UiO-66 is more 
competitive in visible distinguishability than the "turn-off”[ $\left[\mathrm{Cu}_{4} \mathrm{I}(\mathrm{TIPE})_{3}\right] \cdot 3 \mathrm{I}$ [10].

\subsection{Mechanism analysis}

To analyze the sensing phenomenon of FS@UiO-66 towards fluoride, the FTIR spectra before and after immersed in different anion solutions were characterized. As shown in Fig.9, it is obvious that the intensity of the peak at $1018 \mathrm{~cm}^{-1}$ turns weak and the peak at $1643 \mathrm{~cm}^{-1}(\mathrm{C}=\mathrm{O})$ almost entirely disappears after being immersed in fluoride solution.Other anions cannot lead to the similar phenomenon. Theseresultsreflect that the FS molecule has run away from the material into the solution, which directly leads to the bright yellowish green fluorescence.

To explain this phenomenon, PXRD patterns of samples before and after contacted with anions were characterized. As shown in Fig.10, the diffraction peaks almost disappear after being immersed in fluoride solution, indicating that fluoride in aqueous solution can cause the collapse of FS@UiO-66 framework. Herein, to understand this collapse, XPS patterns for FS@UiO-66 before and after contacted with fluoride were further performed. As shown in Fig. 11a, there is a new peak of $\mathrm{F}$ 1s in the pattern of FS@UiO-66 after being immersed in fluoride solution (F-FS@UiO-66), demonstrating the existence of fluoride in the sample. The peaks of Zr $3 \mathrm{~d}_{3 / 2}$ and $3 \mathrm{~d}_{5 / 2}$ shift from $184.68 \mathrm{eV}$ and $182.38 \mathrm{eV}$ to $184.92 \mathrm{eV}$ and $182.52 \mathrm{eV}$ (Fig. 11b) respectively, which may be attributed to the combination of zirconium ion and fluoride[41-42].Therefore, we suggest that fluoride in aqueous solution can lead to the collapse of the FS@UiO-66 framework and then the release of the fluorescent molecule, as shown in Scheme 1. This detection mechanism is similar to that in some 
previous works [17, 43-45].

\subsection{Fluorescence response towardsfluoroorganics}

Furthermore, some fluoroorganicsincluding fluorobenzenes, 8-fluoroquinoline, 2-ethoxy-5-fluoro-1H-pyrimidin-4-one and 5-fluorouracilwere selected as sensing targets to evaluate the sensing ability of FS@UiO-66. As shown in Fig. 12, these selected fluoroorganicshave negligible influence on the fluorescence intensity.However, fluoride in DMF can induce obvious fluorescence enhancement. These results further indicate that FS@UiO-66 is a highly selective sensor for fluoride.

\section{Conclusions}

In summary, fluorescein sodium was successfully incorporated into the Zr-based MOF framework (UiO-66). The systematic investigation demonstrates that the resulting FS@UiO-66 can exhibit high selectivity and fast responseability in sensing fluoride, which is mainly due to the FS molecule release from the framework caused by attack of fluoride. This work demonstrates that MOF sensor can be rationally designed via proper selection of organic fluorescence molecule and MOF framework.

\section{Acknowledgements}

Financial supports by National Science Foundation of China (No. U1332107), Basic Condition Platform Construction Program of Shanxi Province(No. 2012091019), Science and Technology Plan of Jincheng City(No. 201501004-18) and Key Research, Development Plan of Shanxi Province (No. 201603D321017) and Doctoral Scientific Research Foundation of Taiyuan University of Science and 
Technology (No. 20162012) are greatly appreciated.

\section{References}

[1] S. Jagtap, M.K. Yenkie, N. Labhsetwar, S. Rayalu,Fluoride in drinking water and defluoridation of water, Chem. Rev. 112 (2012) 2454-2466.

[2] Y. Nie, C. Hu, C. Kong,Enhanced fluoride adsorption using Al(III) modified calcium hydroxyapatite, J. Hazard. Mater. 233-234 (2012) 194-199.

[3] P.D.C. Dietzel, P.A. Georgiev, J. Eckert, R. Blom, T. Strassle, T. Unruh, Interaction of hydrogen with accessible metal sites in the metal-organic frameworks $\mathrm{M}_{2}$ (dhpt) (CPO-27-M; M=Ni, Co, Mg), Chem. Commun. 46 (2010) 4962-4964.

[4] F. Gandara, H. Furukawa, S. Lee, O.M. Yaghi, High methane storage capacity in aluminum metal-organic frameworks, J. Am. Chem. Soc. 136 (2014) 5271-5274.

[5] J.-R. Li, J. Sculley, H.-C.Zhou, Metal-organic frameworks for separations, Chem. Rev. 112 (2012) 869-932.

[6] L.E. Kreno, K. Leong, O.K. Farha, M. Allendorf, R.P. Van Duyne, J.T. Hupp, Metal-organic framework materials as chemical sensors, Chem. Rev. 112 (2012) 1105-1125.

[7] Y. Cui, Y. Yue, G. Qian, B. Chen, Luminescent functional metal-organic frameworks, Chem. Rev. 112 (2012) 1126-1162.

[8] P. Horcajada, R. Gref, T. Baati, P.K. Allan, G. Maurin, P. Couvreur, G. Férey, R.E. Morris, C. Serre, Metal-organic frameworks in biomedicine, Chem. Rev. 112 (2012) 1232-1268.

[9] H. Huang, W. Zhang, F. Yang, B. Wang, Q. Yang, Y. Xie, C. Zhong, J.-R. Li, 
Enhancing $\mathrm{CO}_{2}$ adsorption and separation ability of $\mathrm{Zr}(\mathrm{IV})$-based metal-organic frameworks through ligand functionalization under the guidance of the quantitative structure-properties relationship model, Chem. Eng. J. 289 (2016) 247-253.

[10] Y. Guo, X. Feng, T. Han, S. Wang, Z. Lin, Y. Dong, B. Wang, Tuning the luminescence of metal-organic frameworks for detection of energetic heterocyclic compounds, J. Am. Chem. Soc. 136 (2014) 15485-15488.

[11] B. Wang, X.-L.Lv, D. Feng, L.-H.Xie, J. Zhang, M. Li, Y. Xie, J.-R. Li, H.-C. Zhou, Highly stable $\mathrm{Zr}(\mathrm{IV})$-based metal-organic frameworks for the detection and removal of antibiotics and organic explosives in water, J. Am. Chem. Soc. 138 (2016) 6204-6216.

[12] Y. Cui, R. Song, J. Yu, M. Liu, Z. Wang, C. Wu, Y. Yang, Z. Wang, B. Chen, G. Qian, Dual-emitting MOF Ddye composite for ratiometric temperature sensing, Adv. Mater. 27 (2015) 1420-1425.

[13] D. Rambabu, Pooja, C.P. Pradeep, A. Dhir, A cytochorme C encapsulated metal organic framework as a bio-material for sulfate ion recognition, J. Mater. Chem. A 2 (2014) 8628-8631.

[14] W. Cho, H.J. Lee, G. Choi, S. Choi, M. Oh, Dual changes in conformation and optical properties of fluorophores within a metal-organic framework during framework construction and associated sensing event, J. Am. Chem. Soc. 136 (2014) 12201-12204.

[15] D. Yan, Y. Tang, H. Lin, D. Wang, Tunable two-color luminescence and host-guest energy transfer of fluorescent chromophores encapsulated in metal-organic 
frameworks, Sci. Rep. 4 (2014) 4337.

[16] T.-T. Han, J. Yang, Y.-Y.Liu, J.-F. Ma, Rhodamine 6G loaded zeoliticimidazolate framework-8 (ZIF-8) nanocomposites for highly selective luminescent sensing of $\mathrm{Fe}^{3+}$, $\mathrm{Cr}^{6+}$ and aniline, Micropor. Mesopor.Mater. 228 (2016) 275-288.

[17] J. Deng, W. Ma, P. Yu, L. Mao, Colorimetric and fluorescent dual mode sensing of alcoholic strength in spirit samples with stimuli-responsive infinite coordination polymers, Anal. Chem. 87 (2015) 6958-6965.

[18] X. Zhu, J. Gu, J. Yang, Z. Wang, Y. Li, L. Zhao, W. Zhao, J. Shi, Zr-based metal-organic frameworks for specific and size-selective enrichment of phosphoptides with simultaneous exclusion of proteins, J. Mater. Chem. C 3 (2015) 4242-4248.

[19] H.R. Abid, H. Tian, H.-M.Ang, M.O. Tade, C.E. Buckley, S. Wang, NanosizeZr-metal organic framework (UiO-66) for hydrogen and carbon dioxide storage, Chem. Eng. J. 187 (2012) 415-420.

[20] P.S. Bárcia, D. Guimarães, D.A.P. Mendas, J.A.C. Silva, V. Guillerm, H. Chevreau, C. Serre, A.E. Rodrigues, Reverse shape selectivity in the adsorption of hexane and xylene isomers in MOF UiO-66, Micropor. Mesopor.Mater. 139 (2011) 67-73.

[21] J. Aguilera-Sigalat, A. Fox-Charles, D. Bradshaw, Direct photo-hydroxylation of the Zr-based framework UiO-66, Chem. Commun. 50 (2014) 15453-15456.

[22] A. Shahat, H.M.A. Hassan, H.M.E. Azzazy, Optical metal-organic framework sensor for selective discrimination of some toxic metal ions in water, Anal.Chim.Acta. 793 (2013) 90-98. 
[23] H. Huang, J.-R. Li, K. Wang, T. Han, M. Tong, L. Li, Y. Xie, Q. Yang, D. Liu, C. Zhong, An insitu self-assembly template strategy for the preparation of hierarchical-pore metal-organic frameworks, Nat. Commun. 6 (2015) 8847.

[24] J.H. Cavka, S. Jakobsen, U. Olsbye, N. Guillou, C. Lamberti, S. Bordiga, K.P. Lillerud, A new zirconium inorganic building brick forming metal organic frameworks with exceptional stability, J. Am. Chem. Soc. 130 (2008) 13850-13851.

[25] Y. Lu, B. Yan, Luminescent lanthanide barcodes based on postsynthetic modified nanoscale metal-organic frameworks, J. Mater. Chem. C 2 (2014) 7411-7416.

[26] Y. Yue, A.J. Binder, R. Song, Y. Cui, J. Chen, D.K. Hensley, S. Dai, Encapsulation of large dye molecules in hierarchically superstructured metal-organic frameworks, Dalton Tran. 43 (2014) 17893-17898.

[27] L. Valenzano, B. Civalleri, S. Chavan, S. Bordiga, M.H. Nilsen, S. Jakobsen, K.P. Lillerud, C. Lamberti, Disclosing the complex structure of UiO-66 metal organic framework: a synergic combination of experiment and theory, Chem. Mater. 23 (2011) 700-718.

[28] L. Zhang, Y. Jian, J. Wang, C. He, X. Li, T. Liu, C. Duan, Post-modification of a MOF through a fluorescent-labeling technology for the selective sensing and adsorption of $\mathrm{Ag}^{+}$in aqueous solution, Dalton Trans. 41 (2012) 10153-10155.

[29] J. Yang, Y. Dai, X. Zhu, Z. Wang, Y. Li, Q. Zhuang, J. Shi, J. Gu, Metal-organic frameworks with inherent recognition sites for selective phosphate sensing through their coordination-induced fluorescence enhancement effect, J. Mater. Chem. A 3 (2015) 7445-7452. 
[30] L.L. Wu, Z. Wang, S.N. Zhao, X. Meng, X.Z. Song, J. Feng, S.Y. Song, H.J. Zhang, A metal-organic framework/DNA hybrid system as a novel fluorescent biosensor for mercury(II) ion detection, Chem. Eur. J. 22 (2016) 477-480.

[31] J.N. Hao, B. Yan, A water-stable lanthanide-functionalized MOF as a highly selective and sensitive fluorescent probe for $\mathrm{Cd}^{2+}$, Chem. Commun. 51 (2015) 7737-7740.

[32] H. Tavallali, G. Deilamy-Rad, M. Tabandeh, A selective detection of fluoride ions in DMSO by fluorescent and colorimetry competition assays based on 4-bromo-2,6-bis-(hydroxymethyl)phenol, Chin. Chem. Lett. 22 (2011) 193-196.

[33] G. Li, W.T. Gong, J.W. Ye, Y. Lin, G.L. Ning, Unprecedented intramolecular cyclization of pyridinium to pyrido[1,2-a]benzimidazole: a novel chemodosimeter for fluoride ions, Tetrahedron Lett. 52 (2011) 1313-1316.

[34] L. Fu, F.L. Jiang, D. Fortin, P.D. Harvey, Y. Liu, A reaction-based chromogenic and fluorescent chemodosimeter for fluoride anions, Chem. Commun. 47 (2011) 5503-5505.

[35] R.C. Mulrooney, N. Singh, N. Kaur, J.F. Callan, An “off-on” sensor for fluoride using luminescent CsSe/ZnS quantum dots, Chem. Commun. 6 (2009) 686-688.

[36] H. Chen, P.X. Liu, S.P. Zhuo, X. Meng, Z.Y. Zhou, H.N. Wang, A [Cu $\left.\mathrm{Cu}_{4}\right]$ cluster based metal-organic framework to detect $\mathrm{F}^{-}$ions, Inorg. Chem. Commun. 63 (2016) 69-73.

[37] J.M. Zhou, W. Shi, N. Xu, P. Cheng, Highly selective luminescent sensing of fluoride and organic small-molecule pollutants based on novel lanthanide 
metal-organic frameworks, Inorg. Chem. 52 (2013) 8082-8090.

[38] K.L. Wong, G.L. Law, Y.Y. Yang, W.T. Wong, A highly porous luminescent terbium-organic framework for reversible anion sensing, Adv. Mater. 18 (2006) 1051-1054.

[39] F.M. Hinterholzinger, B. Rühle, S. Wuttke, K. Karaghiosoff, T. Bein, Highly sensitive and selective fluoride detection in water through fluorophore release from a metal-organic framework, Sci. Rep. 3 (2013) 2562.

[40] B. Chen, L. Wang, F. Zapata, G. Qian, E.B. Lobkovsky, A luminescent microporous metal-organic framework for the recognition and sensing of anions, J. Am. Chem. Soc. 130 (2008) 6718-6719.

[41] Y. Takahashi, D.A. Pacheco Tanaka, H. Matsunaga, T.M. Suzuki, Fluorometric detection of fluoride ion by ligand exchange reaction with 3-hydroxyflavone coordinated to a zirconium (IV)-EDTA complex, J. Chem. Soc., Perkin Trans. 2 (2002) 759-762.

[42] S.D. Wolter, J.R. Piascik, B.R. Stoner, Characterization of plasma fluorinated zirconia for dental applications by X-ray photoelectron spectroscopy, Appl. Surf. Sci. 257 (2011) 10177-10182.

[43] K.S. Asha, R. Bhattacharjee, S. Mandal, Complete transmetalation in a metal-organic framework by metal ion metathesis in a single crystal for selective sensing of phosphate ions in aqueous media, Angew. Chem. Int. Ed. 55 (2016) 11528-11532.

[44] Z.P. Wang, B. Hu, X.H. Qi, N.N. Shen, X.Y. Huang, Microwave-assisted 
ionothermal synthesis of a water-stable Eu-coordination polymer: a $\mathrm{Ba}^{2+}$ ion detectorand fluorescence thermometer, Dalton Tran. 45 (2016) 8745-8752.

[45] X. Lin, F. Luo, L. Zheng, G. Gao, Y. Chi, Fast, sensitive, and selective ion-triggered disassembly and release based on tris(bipyridine)ruthenium(II)functionalized metal-organic frameworks, Anal. Chem. 87 (2015) 4864-4870. 


\section{Figure captions}

Fig. 1.The molecular structure of fluorescein sodium.

Fig. 2.The PXRD patterns (a) and $\mathrm{N}_{2}$ adsorption-desorption isothermals (b) of the MOFs.

Fig. 3.The FTIR spectra of UiO-66, FS@UiO-66 and FS molecule.

Fig. 4.Fluorescence microscopic images of FS@UiO-66 under white light (a) and UV light (b), respectively.

Fig. 5.Excitation and emission spectra of FS@UiO-66 (a); PL intensity at 537 nm with different immersion time of solid-state FS@UiO-66 (b).

Fig. 6.Images of FS@UiO-66 in different anion aqueous solutions $\left(10^{-2} \mathrm{M}\right)$ at natural light (a) and UV=365 nm (b); PL spectra of the upper clear part in different anion solutions $\left(10^{-2} \mathrm{M}\right)(\mathrm{c})$.

Fig. 7.Effect of co-exist anions on the sensing performance of FS@UiO-66 (the concentrations of all the anions solutions are $10^{-2} \mathrm{M}$ ).

Fig. 8.The intensity at $520 \mathrm{~nm}$ in PL spectra of the upper clear part at different immersion time (a); PL spectra of the upper clear part in fluoride solutions with different concentrations (b).

Fig. 9.FTIR spectra of FS@UiO-66 before and after immersed in different anion aqueous solutions $\left(10^{-2} \mathrm{M}\right)$.

Fig. 10.PXRD patterns of FS@UiO-66 before and after immersed in different anion aqueous solutions $\left(10^{-2} \mathrm{M}\right)$.

Fig. 11.XPS patterns (a) and Zr 3d patterns (b) for FS@UiO-66 before and after immersed in fluoride solution $\left(10^{-2} \mathrm{M}\right)$.

Fig. 12.The fluorescence intensity towards different fluorine species.

Table 1.Comparison of the detection limit of FS@UiO-66 with other sensors.

Scheme 1.Schematic illustration of sensing mechanism for fluoride in FS@UiO-66. 
Figure 1

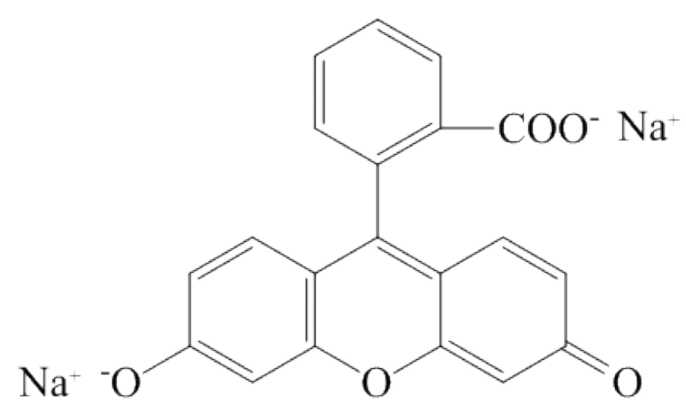


Figure 2
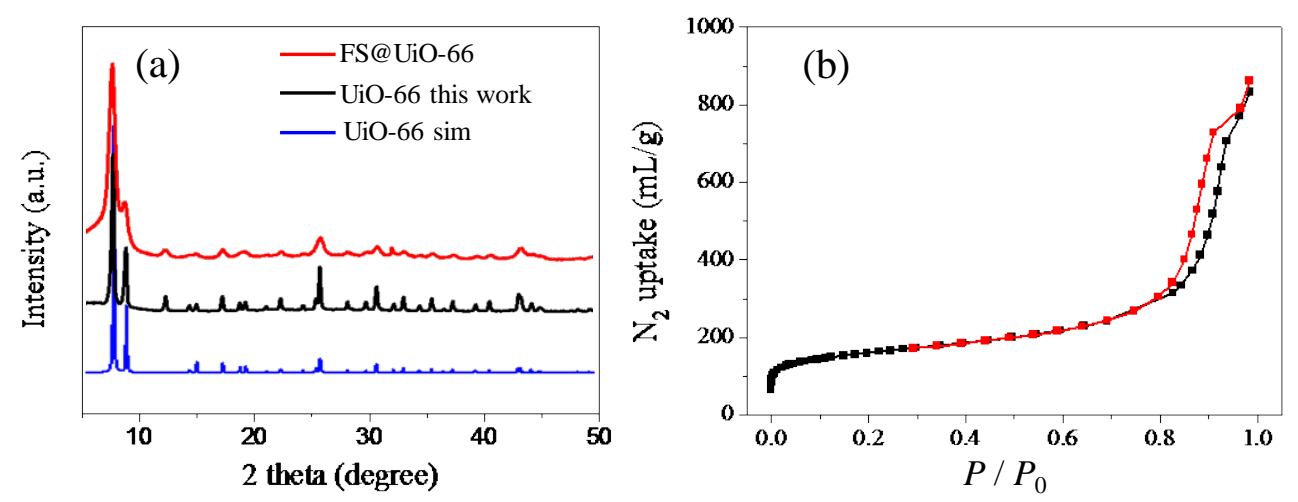
Figure 3

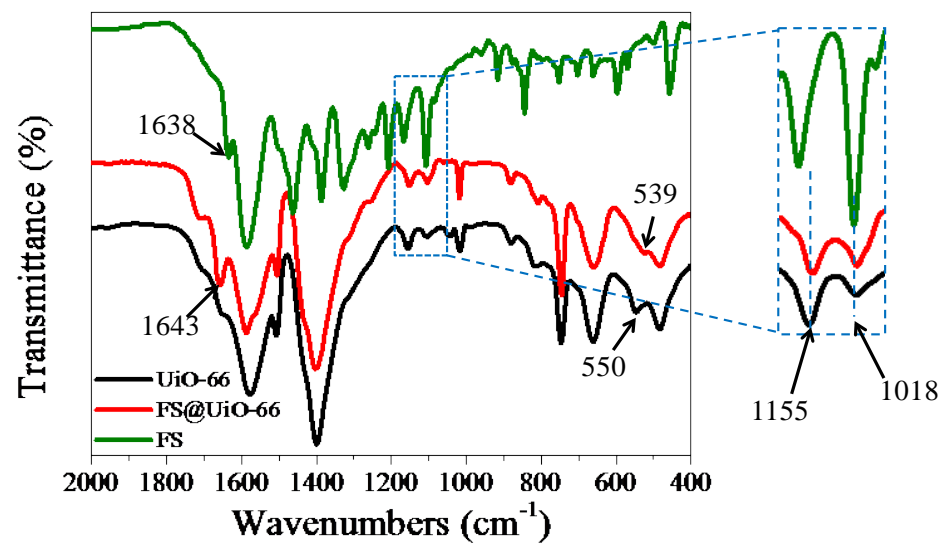


Figure 4
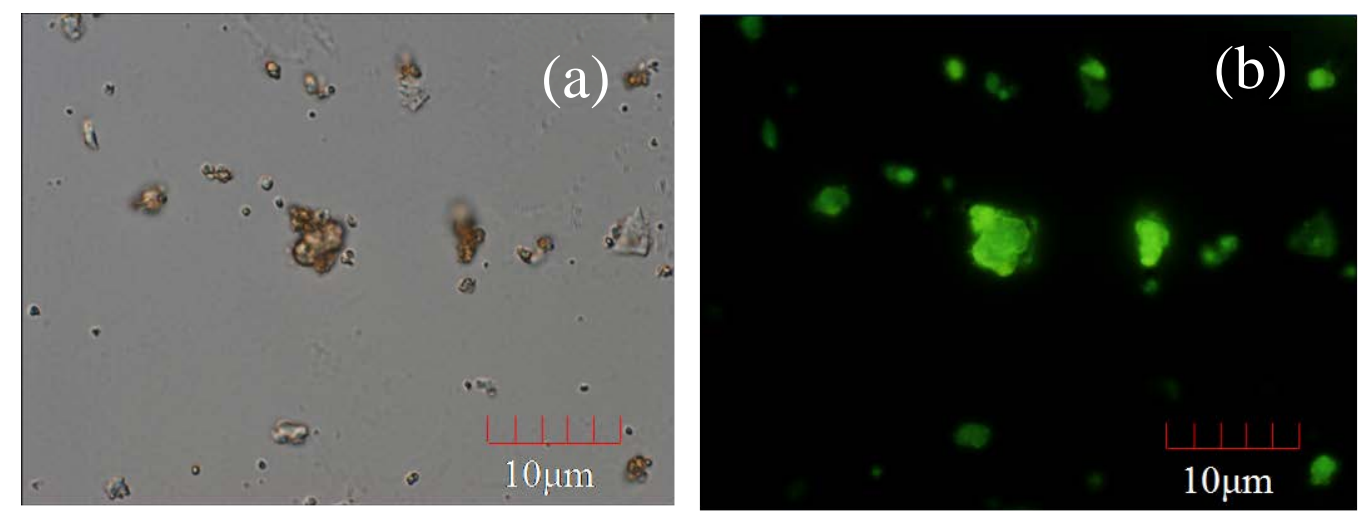
Figure 5
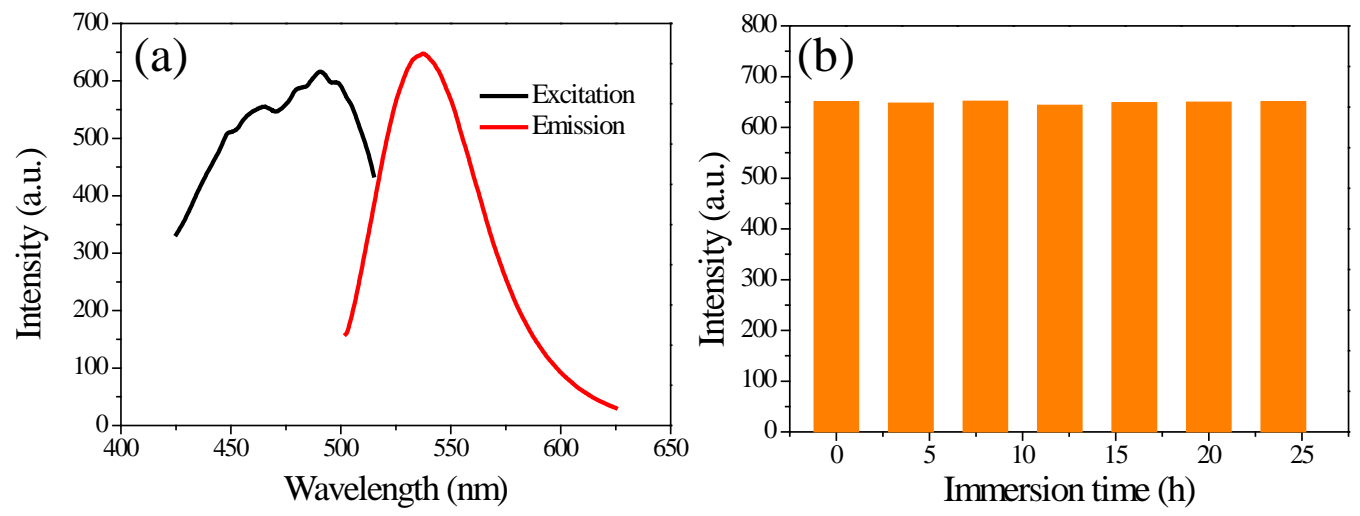
Figure 6
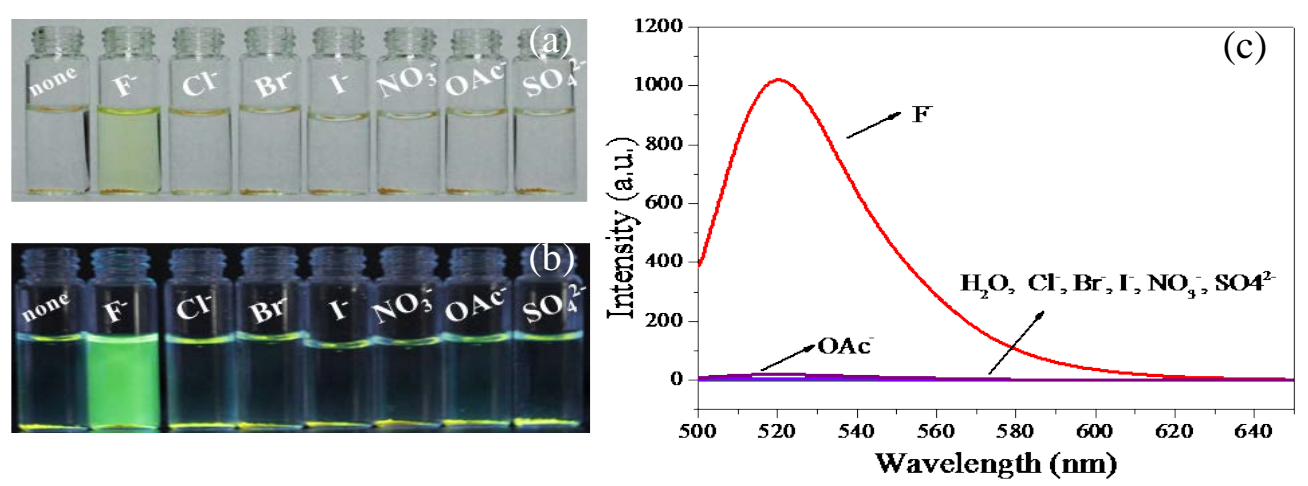
Figure 7

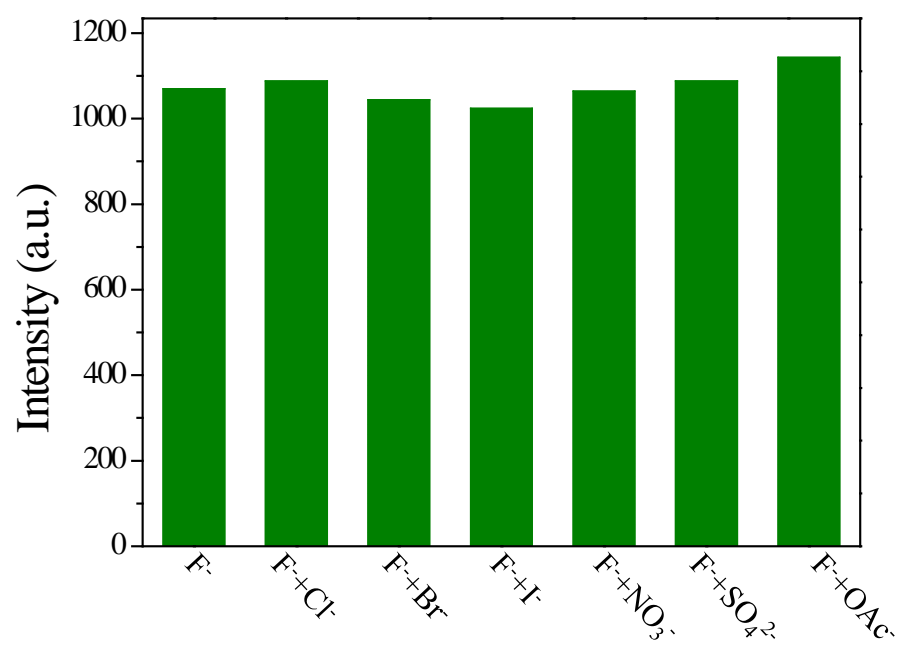


Figure 8
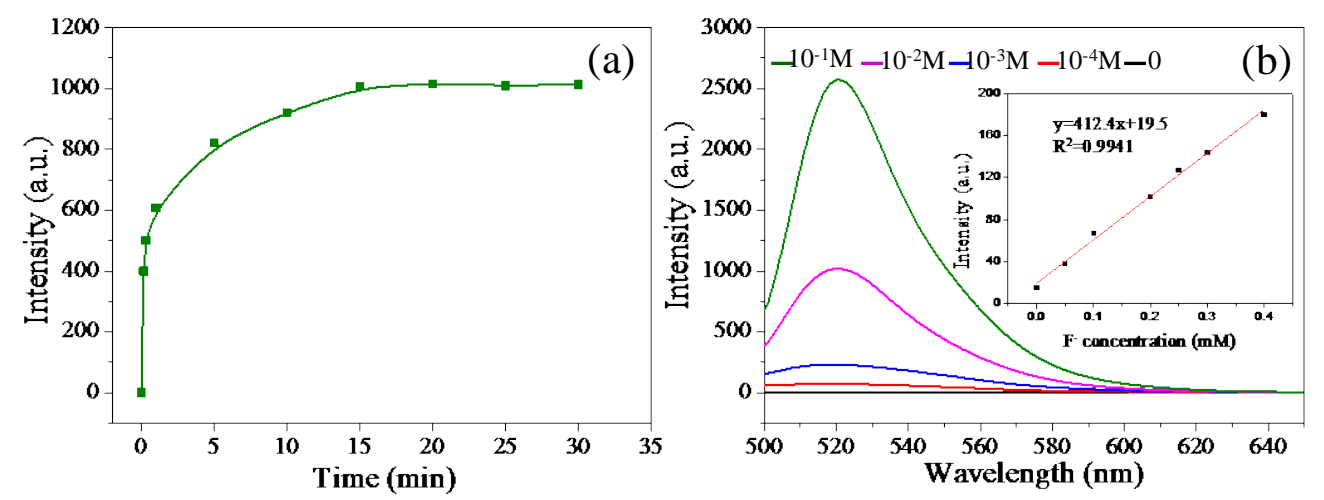
Figure 9

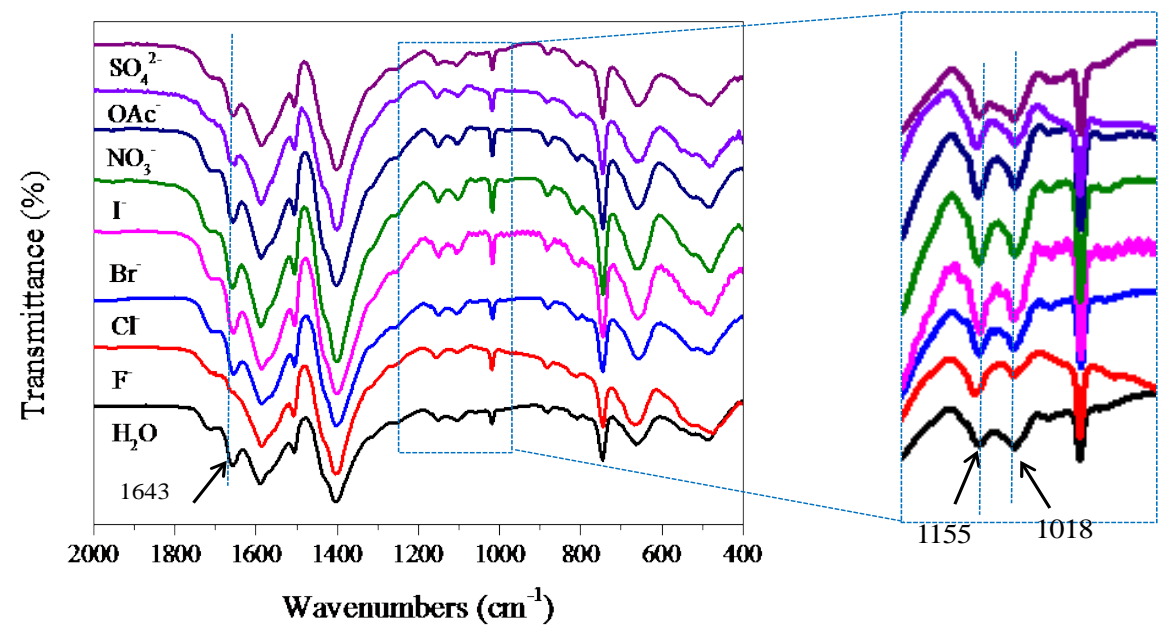


Figure 10

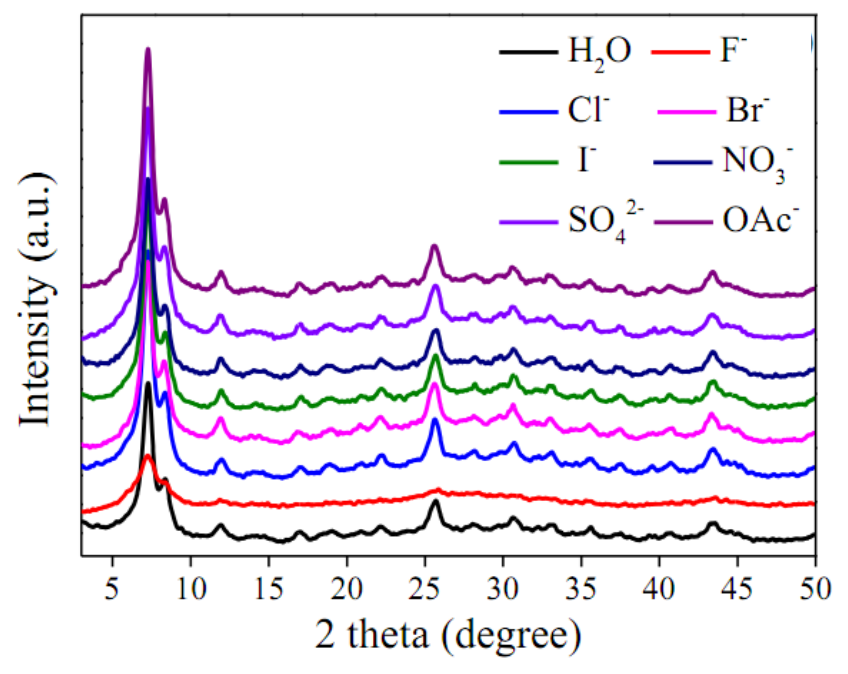


Figure 11
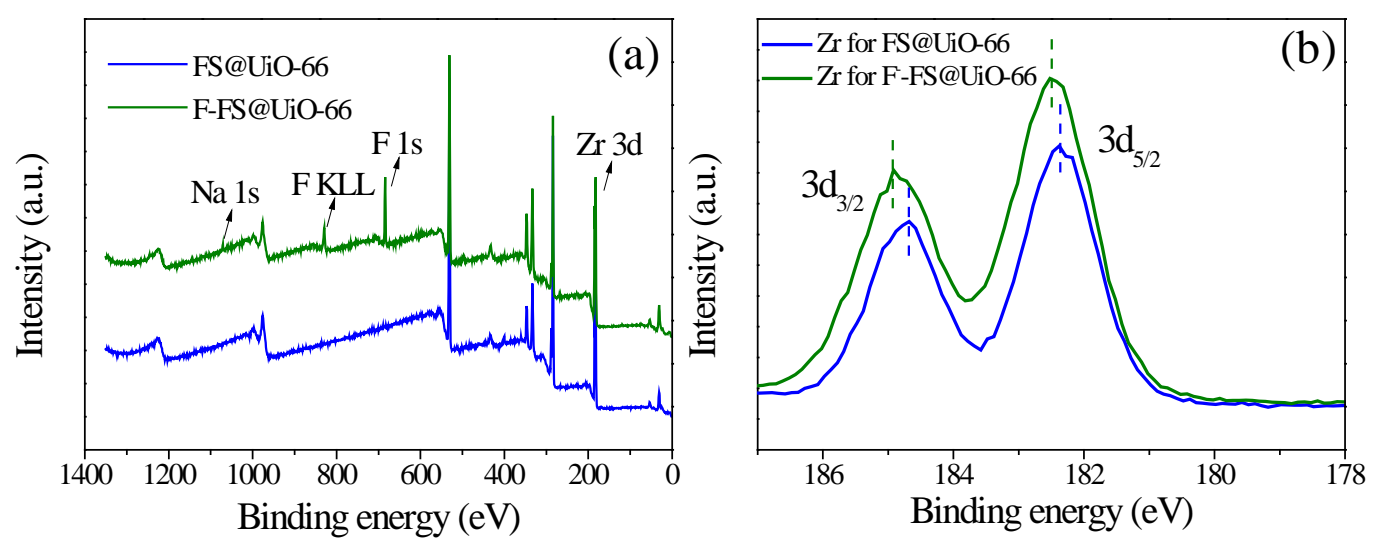
Figure 12

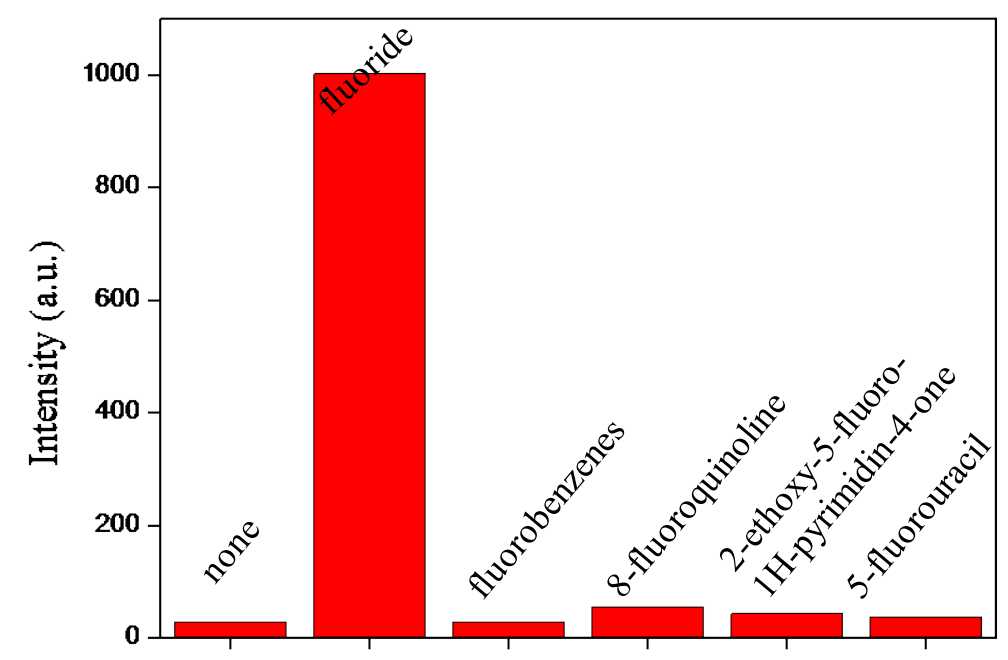

Fluorine species 
Table 1 Comparison of the detection limit of FS@UiO-66 with other sensors

\begin{tabular}{|c|c|c|c|}
\hline Sensor & $\begin{array}{l}\text { Detection limit } \\
\qquad(\mathrm{mM})\end{array}$ & Solvent & Ref. \\
\hline 4-bromo-2,6-bis(hydroxymethyl)phenol & $0.9 \times 10^{-3}$ & DMSO & [32] \\
\hline Aminophenylpyridinium & $2.72 \times 10^{-3}$ & $\mathrm{CH}_{3} \mathrm{CN}$ & [33] \\
\hline BODIPY dye-containing chemodosimeter & $0.0674 \times 10^{-3}$ & Acetone & [34] \\
\hline CdSe/ZnS QD & $74.0 \pm 0.11$ & $\mathrm{CHCl}_{3}$ & [35] \\
\hline$\left[\mathrm{Cu}_{4} \mathrm{I}(\mathrm{TIPE})_{3}\right] \cdot 3 \mathrm{I}$ & $2.11 \times 10^{-3}$ & $\mathrm{H}_{2} \mathrm{O}$ & [36] \\
\hline $\mathrm{Eu}_{2}(\mathrm{bpdc})(\mathrm{bdc})_{2}\left(\mathrm{H}_{2} \mathrm{O}\right)$ & - & $\mathrm{H}_{2} \mathrm{O}$ & [37] \\
\hline $\mathrm{Tb}_{2}(\mathrm{bpdc})(\mathrm{bdc})_{2}\left(\mathrm{H}_{2} \mathrm{O}\right)$ & - - & $\mathrm{H}_{2} \mathrm{O}$ & [37] \\
\hline$\left[\mathrm{Tb}(\text { Mucicate })_{1.5}\left(\mathrm{H}_{2} \mathrm{O}\right)_{2}\right] \cdot 5 \mathrm{H}_{2} \mathrm{O}$ & - & HEPES & [38] \\
\hline Modified $\mathrm{NH}_{2}$-MIL-101(Al) & - & $\mathrm{H}_{2} \mathrm{O}$ & [39] \\
\hline MOF-76 & - - & $\mathrm{MeOH}$ & {$[40]$} \\
\hline FS@UiO-66 & $4.4 \times 10^{-3}$ & $\mathrm{H}_{2} \mathrm{O}$ & this work \\
\hline
\end{tabular}


Scheme 1

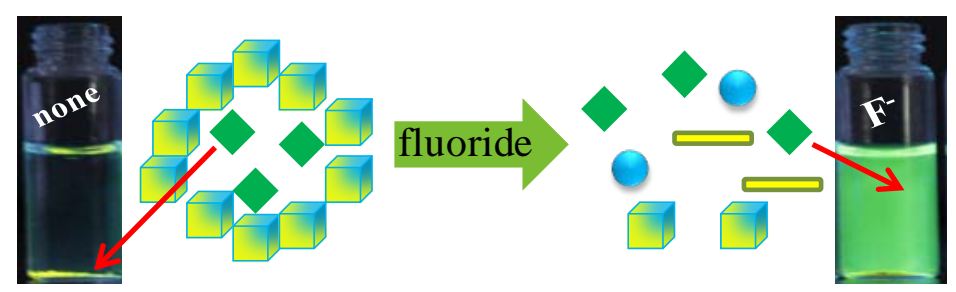

metal ion $\Longleftarrow$ ligand $\triangle$ FS molecule $\square$ MOF particle 